ANNALES

POLONICI MATHEMATICI

$86.3(2005)$

\title{
Explicit extension maps in intersections of non-quasi-analytic classes
}

\author{
by JeAn Schmets (Liège) and MAnuel VAldivia (Valencia)
}

\author{
To our friend Klaus Dieter Bierstedt \\ on the occasion of his 60th birthday
}

\begin{abstract}
We deal with projective limits of classes of functions and prove that: (a) the Chebyshev polynomials constitute an absolute Schauder basis of the nuclear Fréchet spaces $\mathcal{E}_{(\mathfrak{M})}\left([-1,1]^{r}\right)$; (b) there is no continuous linear extension map from $\Lambda_{(\mathfrak{M})}^{(r)}$ into $\mathcal{B}_{(\mathfrak{M})}\left(\mathbb{R}^{r}\right)$; (c) under some additional assumption on $\mathfrak{M}$, there is an explicit extension map from $\mathcal{E}_{(\mathfrak{M})}\left([-1,1]^{r}\right)$ into $\mathcal{D}_{(\mathfrak{M})}\left([-2,2]^{r}\right)$ by use of a modification of the Chebyshev polynomials. These results extend the corresponding ones obtained by Beaugendre in [1] and [2].
\end{abstract}

1. Introduction. It is well known that there is no continuous linear extension map from $\omega$ into $\mathcal{C}^{\infty}(\mathbb{R})$. In 1961, Mityagin [4] proved that (a) $\mathcal{C}^{\infty}([-1,1])$ is a Fréchet nuclear space in which the Chebyshev polynomials constitute an absolute Schauder basis, and (b) there is a continuous linear extension map from $\mathcal{C}^{\infty}([-1,1])$ into $\mathcal{C}^{\infty}(\mathbb{R})$ by means of a modification of the Chebyshev polynomials.

Since then, (non-) existence of continuous linear extension maps has been extensively studied in the case of non-quasi-analytic classes. For instance, we mention the results by Petzsche [5] about the Borel case, i.e. the case $n=1$ and $K=\{0\}$.

Recently Beaugendre [1], [2] has obtained similar results in the case of some projective limits of such classes.

In this paper we deal with these questions in a more general setting, i.e. in the case of countable intersections of classes of functions defined by means of matrices of positive elements.

2000 Mathematics Subject Classification: 26E10, 46E10.

Key words and phrases: ultradifferentiable functions, Beurling classes, Schauder basis, Chebyshev polynomials, extension maps.

Research of M. Valdivia partially supported by MCYT and FEDER Project BFM $2002-01423$. 
After having obtained some inequalities, we introduce in Section 4 the matrices $\mathfrak{m}$ and $\mathfrak{M}$ that allow us to define for instance the spaces $\mathcal{B}_{\left(\boldsymbol{M}_{j}\right)}(\Omega)$, $\mathcal{E}_{\left(\boldsymbol{M}_{j}\right)}\left([-1,1]^{r}\right)$ and $\Lambda_{\left(\boldsymbol{M}_{j}\right)}^{(r)}$ by use of the rows of $\mathfrak{M}$, and next their projective $\operatorname{limits} \mathcal{B}_{(\mathfrak{M})}(\Omega), \mathcal{E}_{(\mathfrak{M})}\left([-1,1]^{r}\right)$ and $\Lambda_{(\mathfrak{M})}^{(r)}$.

We then prove that for every $r \in \mathbb{N}$,

(a) $\mathcal{E}_{(\mathfrak{M})}\left([-1,1]^{r}\right)$ is a Fréchet nuclear space in which the Chebyshev polynomials constitute an absolute Schauder basis;

(b) there is no continuous linear extension map from $\Lambda_{(\mathfrak{M})}^{(r)}$ into $\mathcal{B}_{(\mathfrak{M})}\left(\mathbb{R}^{r}\right)$.

Under some additional assumption on $\mathfrak{M}$, we also obtain the existence of a continuous linear extension map from $\mathcal{E}_{(\mathfrak{M})}\left([-1,1]^{r}\right)$ into $\mathcal{D}_{(\mathfrak{M})}\left([-2,2]^{r}\right)$, hence into $\mathcal{B}_{(\mathfrak{M})}\left(\mathbb{R}^{r}\right)$.

Finally, we prove that these results extend the corresponding ones obtained by Beaugendre in [1] and [2].

\section{An auxiliary inequality}

Proposition 2.1. Let $r, m \in \mathbb{N}, \Omega$ be an open subset of $\mathbb{R}^{r}$ and $f \in$ $\mathcal{C}^{m}(\Omega)$. For every $s \in\{1, \ldots, r\}$, let moreover $\omega_{s}$ be an open subset of $\mathbb{R}$ and $g_{s} \in \mathcal{C}^{m}\left(\omega_{s}\right)$ be a real function such that

$$
\left(g_{1}\left(t_{1}\right), \ldots, g_{r}\left(t_{r}\right)\right) \in \Omega, \quad \forall\left(t_{1}, \ldots, t_{r}\right) \in \omega:=\omega_{1} \times \cdots \times \omega_{r},
$$

and

$$
M:=\sup _{1 \leq s \leq r} \sup _{0 \leq l \leq m}\left\|g_{s}^{(l)}\right\|_{\omega_{s}}<\infty .
$$

Then for every $\alpha \in \mathbb{N}_{0}^{r}$ such that $0<|\alpha| \leq m$, there are explicit functions $A_{\beta}$ on $\omega$ such that

$$
\frac{1}{\alpha !}\left(f\left(g_{1}, \ldots, g_{r}\right)\right)^{(\alpha)}(t)=\sum_{0 \neq \beta \leq \alpha} A_{\beta}(t) \frac{1}{\beta !} f^{(\beta)}\left(g_{1}\left(t_{1}\right), \ldots, g_{r}\left(t_{r}\right)\right)
$$

and $\sum_{0 \neq \beta \leq \alpha}\left|A_{\beta}(t)\right| \leq(1+M)^{|\alpha|}$ for every $t \in \omega$. Therefore for every $\alpha \in \mathbb{N}_{0}^{r}$ such that $0 \leq|\alpha| \leq m$ and $t \in \omega$,

$$
\frac{1}{\alpha !}\left|\left(f\left(g_{1}, \ldots, g_{r}\right)\right)^{(\alpha)}(t)\right| \leq(1+M)^{|\alpha|} \sup _{0 \leq \beta \leq \alpha} \frac{1}{\beta !}\left|f^{(\beta)}\left(g_{1}\left(t_{1}\right), \ldots, g_{r}\left(t_{r}\right)\right)\right| .
$$

Proof. Once the first assertion is established, the consequence is immediate since the case $\alpha=0$ is trivial.

The case $r=1$ is a direct consequence of the Faà di Bruno formula (cf. [7]) stating that, for every $s \in\{1, \ldots, m\}$,

$$
\frac{\left(f\left(g_{1}\right)\right)^{(s)}(t)}{s !}=\sum_{k=1}^{s} \frac{f^{(k)}\left(g_{1}(t)\right)}{k !} A_{k}(t), \quad \forall t \in \omega_{1},
$$


where

$$
A_{k}(t)=\sum_{\substack{k_{1}+\cdots+k_{s}=k \\ k_{1}+2 k_{2}+\cdots+s k_{s}=s}} \frac{k !}{k_{1} ! \cdots k_{s} !}\left(\frac{g_{1}^{(1)}(t)}{1 !}\right)^{k_{1}} \ldots\left(\frac{g_{1}^{(s)}(t)}{s !}\right)^{k_{s}}
$$

with

$$
\sum_{\substack{k_{1}+\cdots+k_{s}=k \\
k_{1}+2 k_{2}+\cdots+s k_{s}=s}} \frac{k !}{k_{1} ! \ldots k_{s} !}=\left(\begin{array}{c}
s-1 \\
k-1
\end{array}\right) .
$$

It is indeed sufficient to note that

$$
\sum_{k=1}^{s}\left|A_{k}(t)\right| \leq \sum_{k=1}^{s} M^{k}\left(\begin{array}{l}
s-1 \\
k-1
\end{array}\right)=M(1+M)^{s-1} \leq(1+M)^{s} .
$$

To conclude by induction, we just have to prove that if the property is true for $r-1$ with $r \geq 2$, then it is also true for $r$.

Of course we may suppose $\alpha_{1} \neq 0$. For every $t_{1} \in \omega_{1}$ and $x_{2}, \ldots, x_{r} \in \mathbb{R}$ such that $\left(g_{1}\left(t_{1}\right), x_{2}, \ldots, x_{r}\right) \in \Omega$, the case $r=1$ provides

$$
\frac{1}{\alpha_{1} !}\left(f\left(g_{1}, x_{2}, \ldots, x_{r}\right)\right)^{\left(\alpha_{1}\right)}\left(t_{1}\right)=\sum_{\beta_{1}=1}^{\alpha_{1}} A_{\beta_{1}}\left(t_{1}\right) \frac{1}{\beta_{1} !} f^{\left(\beta_{1}^{\prime}\right)}\left(g_{1}\left(t_{1}\right), x_{2}, \ldots, x_{r}\right)
$$

with $\beta_{1}^{\prime}=\left(\beta_{1}, 0, \ldots, 0\right)$ and $\sum_{\beta_{1}=1}^{\alpha_{1}}\left|A_{\beta_{1}}\left(t_{1}\right)\right| \leq(1+M)^{\alpha_{1}}$ for every $t_{1} \in \omega_{1}$.

Now we set $\gamma=\left(\alpha_{2}, \ldots, \alpha_{r}\right)$. In the case $\gamma=0$, we have

$$
\frac{1}{\alpha !}\left(f\left(g_{1}, \ldots, g_{r}\right)\right)^{(\alpha)}(t)=\frac{1}{\alpha_{1} !}\left(f\left(g_{1}, g_{2}\left(t_{2}\right), \ldots, g_{r}\left(t_{r}\right)\right)\right)^{\left(\alpha_{1}\right)}\left(t_{1}\right)
$$

and it suffices to set $A_{\beta}(t)=A_{\beta_{1}}\left(t_{1}\right)$ for every $\beta \in \mathbb{N}_{0}^{r}$ such that $0 \neq \beta \leq \alpha$.

If $\gamma \neq 0$, the commutativity of the derivatives yields

$$
\frac{\left(f\left(g_{1}, \ldots, g_{r}\right)\right)^{(\alpha)}(t)}{\alpha !}=\sum_{\beta_{1}=1}^{\alpha_{1}} \frac{A_{\beta_{1}}\left(t_{1}\right)}{\beta_{1} !} \frac{\left(f^{\left(\beta_{1}^{\prime}\right)}\left(g_{1}\left(t_{1}\right), g_{2}, \ldots, g_{r}\right)\right)^{(\gamma)}\left(t_{2}, \ldots, t_{r}\right)}{\gamma !}
$$

Then we apply the case $r-1$ to get

$$
\begin{aligned}
& \frac{\left(f^{\left(\beta_{1}^{\prime}\right)}\left(g_{1}\left(t_{1}\right), g_{2}, \ldots, g_{r}\right)\right)^{(\gamma)}\left(t_{2}, \ldots, t_{r}\right)}{\gamma !} \\
& =\sum_{0 \neq \eta \leq \gamma} A_{\eta}\left(t_{2}, \ldots, t_{r}\right) \frac{f^{\left(\beta_{1}, \eta\right)}\left(g_{1}\left(t_{1}\right), \ldots, g_{r}\left(t_{r}\right)\right)}{\eta !}
\end{aligned}
$$

with $\sum_{0 \neq \eta \leq \gamma}\left|A_{\eta}\left(t_{2}, \ldots, t_{r}\right)\right| \leq(1+M)^{|\gamma|}$. So the formula is correct if we set

$$
A_{\beta}\left(t_{1}, \ldots, t_{r}\right)= \begin{cases}0 & \text { if } \beta_{1}=0 \text { or }\left(\beta_{2}, \ldots, \beta_{r}\right)=0, \\ A_{\beta_{1}}\left(t_{1}\right) A_{\left(\beta_{2}, \ldots, \beta_{r}\right)}\left(t_{2}, \ldots, t_{r}\right) & \text { otherwise, }\end{cases}
$$


since then

$$
\sum_{0 \neq \beta \leq \alpha}\left|A_{\beta}(t)\right|=\sum_{\beta_{1}=1}^{\alpha_{1}}\left|A_{\beta_{1}}\left(t_{1}\right)\right| \sum_{0 \neq \eta \leq\left(\beta_{2}, \ldots, \beta_{r}\right)}\left|A_{\eta}\left(t_{2}, \ldots, t_{r}\right)\right| \leq(1+M)^{|\alpha|} \text {. - }
$$

3. The Chebyshev polynomials. For every $n \in \mathbb{N}_{0}$, the Chebyshev polynomial $T_{n}$ is the polynomial of degree $n$ on $\mathbb{R}$ which coincides with the function $\cos (n \arccos (x))$ on the interval $[-1,1]$.

The following information about $\left|T_{n}^{(p)}(x)\right|$ will be used. For $T_{0}=\chi_{\mathbb{R}}$, the situation is clear. For every $n \in \mathbb{N}$, the $V$. A. Markov inequality $([6,1.3 .35$ and 1.5.6]) states that

$$
\left|T_{n}^{(p)}(x)\right| \leq T_{n}^{(p)}(1)=\frac{n^{2}}{1} \frac{n^{2}-1^{2}}{3} \cdots \frac{n^{2}-(p-1)^{2}}{2 p-1} \leq \frac{n^{2 p}}{p !}
$$

for every $p \in \mathbb{N}_{0}$ and $x \in[-1,1]$. We need the following slight extension of this inequality.

Proposition 3.1. For every $n \in \mathbb{N}$ and $p \in \mathbb{N}_{0}$, one has

$$
\left|T_{n}^{(p)}(x)\right| \leq e \frac{n^{2 p}}{p !}, \quad \forall x \in\left[-1-\frac{1}{1+n^{2}}, 1+\frac{1}{1+n^{2}}\right] .
$$

Proof. As $T_{n}$ is a polynomial of degree $n$, we only need to consider the case $p \leq n$. Since every $x \in\left[0,1+1 /\left(1+n^{2}\right)\right]$ can be written as $x=y+z$ with $y \in[0,1]$ and $z \in\left[0,1 /\left(1+n^{2}\right)\right]$, the Taylor formula provides the equality $T_{n}^{(p)}(x)=\sum_{k=0}^{n-p} T_{n}^{(p+k)}(y) z^{k} / k$ !, which leads to

$$
\left|T_{n}^{(p)}(x)\right| \leq \sum_{k=0}^{n-p} \frac{n^{2 p}}{(p+k) !} \frac{n^{2 k}}{\left(1+n^{2}\right)^{k}} \frac{1}{k !} \leq \frac{n^{2 p}}{p !} \sum_{k=0}^{\infty} \frac{1}{k !} .
$$

Hence the conclusion follows, since a similar argument works for the elements of the interval $\left[-1-1 /\left(1+n^{2}\right), 0\right]$.

As we will deal with functions defined on subsets of $\mathbb{R}^{r}$, the following considerations will be very useful.

Notation. For every $\gamma \in \mathbb{N}_{0}^{r}, T_{\gamma}$ designates the polynomial defined on $\mathbb{R}^{r}$ by $T_{\gamma}(x)=T_{\gamma_{1}}\left(x_{1}\right) \cdots T_{\gamma_{r}}\left(x_{r}\right)$ for every $x \in \mathbb{R}^{r}$.

Then for every $f \in \mathcal{C}^{\infty}\left([-1,1]^{r}\right), f\left(\cos \left(t_{1}\right), \ldots, \cos \left(t_{r}\right)\right)$ is a periodic $\mathcal{C}^{\infty}$-function on $\mathbb{R}^{r}$. So its Fourier development is

$$
\sum_{\gamma \in \mathbb{N}_{0}^{r}} a_{\gamma}(f) \cos \left(\gamma_{1} t_{1}\right) \cdots \cos \left(\gamma_{r} t_{r}\right)
$$


with

(1) $a_{\gamma}(f)=\frac{1}{2^{r(\gamma)}} \frac{1}{\pi^{r}} \int_{[-\pi, \pi]^{r}} f\left(\cos \left(t_{1}\right), \ldots, \cos \left(t_{r}\right)\right) \cos \left(\gamma_{1} t_{1}\right) \cdots \cos \left(\gamma_{r} t_{r}\right) d t$

where $r(\gamma)$ is the number of components of $\gamma$ which are equal to 0 . Moreover the derivatives of this series converge absolutely and uniformly on $\mathbb{R}^{r}$ to the corresponding derivatives of the function $f\left(\cos \left(t_{1}\right), \ldots, \cos \left(t_{r}\right)\right)$. Therefore we get

$$
f(x)=f\left(x_{1}, \ldots, x_{r}\right)=\sum_{\gamma \in \mathbb{N}_{0}^{r}} a_{\gamma}(f) T_{\gamma}(x)
$$

and the derivatives term by term of this series converge absolutely and uniformly on $[-1,1]^{r}$ to the corresponding derivatives of $f$.

An estimate of the numbers $\left|a_{\gamma}(f)\right|$ can be obtained as follows.

Notation. Given $\gamma \in \mathbb{N}_{0}^{r}$, we set $\gamma^{*}=\left(\gamma_{1}^{*}, \ldots, \gamma_{r}^{*}\right)$ with $\gamma_{s}^{*}=\gamma_{s}$ if $\gamma_{s} \neq 0$ and $\gamma_{s}^{*}=1$ if $\gamma_{s}=0$.

Moreover we set $\gamma^{\alpha}=\left(\gamma_{1}^{*}\right)^{\alpha_{1}} \ldots\left(\gamma_{r}^{*}\right)^{\alpha_{r}}$ for every $\alpha \in \mathbb{N}_{0}^{r}$.

Then, by integration by parts, for every $\alpha \in \mathbb{N}_{0}^{r}$, formula (1) leads to

$$
\left|a_{\gamma}(f)\right|=\frac{1}{2^{r(\gamma)}} \frac{1}{\pi^{r}} \frac{1}{\gamma^{\alpha}}\left|\int_{[-\pi, \pi]^{r}}\left(f\left(\cos \left(t_{1}\right), \ldots, \cos \left(t_{r}\right)\right)\right)^{(\alpha)} *(t) d t\right|
$$

with

$$
*(t)=\left\{\begin{array}{c}
\cos \\
\sin
\end{array}\right\}\left(\gamma_{1} t_{1}\right) \ldots\left\{\begin{array}{l}
\cos \\
\sin
\end{array}\right\}\left(\gamma_{r} t_{r}\right)
$$

hence

$$
\left|a_{\gamma}(f)\right| \leq \alpha ! \frac{2^{r+|\alpha|-r(\gamma)}}{\gamma^{\alpha}} \sup _{0 \leq \delta \leq \alpha} \sup _{x \in[-1,1]^{r}} \frac{\left|f^{(\delta)}(x)\right|}{\delta !}
$$

by Proposition 2.1 .

4. The matrix $\mathfrak{m}$. To any sequence $\boldsymbol{m}=\left(m_{p}\right)_{p \in \mathbb{N}_{0}}$ of positive numbers, we associate as usual the sequence $\boldsymbol{M}=\left(M_{p}\right)_{p \in \mathbb{N}_{0}}$ defined by $M_{p}=$ $m_{0} \cdots m_{p}$ for every $p \in \mathbb{N}_{0}$.

Throughout the paper, $\mathfrak{m}$ designates a matrix

$$
\mathfrak{m}=\left(m_{j, p}\right) \underset{j \in \mathbb{N}}{j \in \mathbb{N}_{0}}
$$

such that the sequences $\boldsymbol{m}_{j}=\left(m_{j, p}\right)_{p \in \mathbb{N}_{0}}$ satisfy the following conditions: for every $j \in \mathbb{N}$,

$\left(\mathfrak{m}_{1}\right) \quad m_{j, 0}=1$ and $m_{j, p} \geq 1$ for every $p \in \mathbb{N}$;

$\left(\mathfrak{m}_{2}\right) \quad m_{j, p} / p \leq m_{j, p+1} /(p+1)$ for every $p \in \mathbb{N}$; in particular the sequence $\left(M_{j, p} / p !\right)_{p \in \mathbb{N}_{0}}$ is increasing and $M_{j, p} \geq p$ ! for every $p \in \mathbb{N}_{0}$;

$\left(\mathfrak{m}_{3}\right) \quad m_{j, p} \geq m_{j+1, p}$ for every $p \in \mathbb{N}_{0}$; 
$\left(\mathfrak{m}_{4}\right) \quad$ for every $A>0$, there is $A_{j}>1$ such that

$$
M_{j+1,2(p+1)} \leq A_{j} A^{-p} p ! M_{j, p}, \quad \forall p \in \mathbb{N}_{0} .
$$

Conditions $\left(\mathfrak{m}_{1}\right)$ and $\left(\mathfrak{m}_{2}\right)$ are standard. Condition $\left(\mathfrak{m}_{3}\right)$ is necessary to introduce the projective limits defined in the next section. Condition $\left(\mathfrak{m}_{4}\right)$ is not standard: it provides links between the spaces defining these projective limits. Let us note at once that it leads to the following two consequences.

Proposition 4.1. For every $j \in \mathbb{N}$ and $B>0$, there is $c>0$ such that $M_{j, p} / p ! \geq c B^{p+1}$ for every $p \in \mathbb{N}_{0}$.

Proof. Indeed, by condition $\left(\mathfrak{m}_{4}\right)$ with $A=B / 4$, we get

$$
\frac{M_{j, p}}{p !} \geq \frac{A^{p}}{A_{j}} \frac{M_{j+1,2(p+1)}}{(p !)^{2}} \geq \frac{A^{p}}{A_{j}}(2 p+1) \frac{(2 p) !}{(p !)^{2}} \geq \frac{(4 A)^{p}}{A_{j}} .
$$

Notation. For every $j \in \mathbb{N}$ and $\gamma \in \mathbb{N}_{0}^{r}$, set $M_{j, \gamma}=M_{j, \gamma_{1}} \cdots M_{j, \gamma_{r}}$.

Proposition 4.2. For every $j, r \in \mathbb{N}$ such that $r \geq 2$, there is a constant $B_{j, r}>1$ such that $M_{j+r-1,|\delta|} \leq B_{j, r}^{|\delta|} M_{j, \delta}$ for every $\delta \in \mathbb{N}_{0}^{r}$.

Proof. When $r=2$, this is equivalent to establishing that, for every $j \in \mathbb{N}$, there is $B_{j}>1$ such that $M_{j+1, p+q} \leq B_{j}^{p+q} M_{j, p} M_{j, q}$ for every $p, q \in \mathbb{N}_{0}$ such that $p \geq q$. For $A=1$, condition $\left(\mathfrak{m}_{4}\right)$ provides a constant $\left.C_{j} \in\right] 0,1\left[\right.$ such that $C_{j} M_{j+1,2(p+1)} \leq p ! M_{j, p}$ for every $p \in \mathbb{N}_{0}$, hence

$$
\begin{aligned}
M_{j, p} M_{j, q} & \geq \frac{C_{j}}{p !} \frac{M_{j+1,2(p+1)}}{(2(p+1)) !}(2(p+1)) ! q ! \geq \frac{C_{j}}{p !} \frac{M_{j+1, p+q}}{(p+q) !}(2 p) ! q ! \\
& \geq C_{j} \frac{M_{j+1, p+q}}{2^{p+q}} \frac{(2 p) !}{p !^{2}} \geq\left(\frac{C_{j}}{2}\right)^{p+q} M_{j+1, p+q} .
\end{aligned}
$$

The case $r \geq 3$ is then immediate:

$$
\begin{aligned}
M_{j+r-1,|\delta|} & \leq B_{j+r-2,2}^{|\delta|} M_{j+r-2, \delta_{1}+\cdots+\delta_{r-1}} M_{j, \delta_{r}} \\
& \leq \cdots \leq\left(B_{j+r-2,2} \cdots B_{j, 2}\right)^{|\delta|} M_{j, \delta_{1}} \cdots M_{j, \delta_{r}} .
\end{aligned}
$$

\section{Some spaces}

The Fréchet spaces $\Lambda_{\left(\boldsymbol{M}_{j}\right)}^{(r)}$ and $\Lambda_{(\mathfrak{M})}^{(r)}$. For every $j, r \in \mathbb{N}$, the Fréchet space $\Lambda_{\left(\boldsymbol{M}_{j}\right)}^{(r)}$ is the vector space of indexed families $\boldsymbol{a}=\left(a_{\gamma}\right)_{\gamma \in \mathbb{N}_{0}^{r}}$ of elements of $\mathbb{K}$ such that

$$
|\boldsymbol{a}|_{j, h}:=\sup _{\gamma \in \mathbb{N}_{0}^{r}} \frac{\left|a_{\gamma}\right|}{h^{|\gamma|} M_{j,|\gamma|}}<\infty, \quad \forall h>0,
$$

endowed with the system of norms $\left\{|\cdot|_{j, 1 / m}: m \in \mathbb{N}\right\}$. 
For every $r \in \mathbb{N}$, the space $\Lambda_{(\mathfrak{M})}^{(r)}$ is then defined as the projective limit of the sequence $\left(\Lambda_{\left(\boldsymbol{M}_{j}\right)}^{(r)}\right)_{j \in \mathbb{N}} ;\left\{|\cdot|_{j, 1 / j}: j \in \mathbb{N}\right\}$ is of course a fundamental system of norms for this space.

The Fréchet spaces $\mathcal{B}_{\left(\boldsymbol{M}_{j}\right)}(\Omega)$ and $\mathcal{B}_{(\mathfrak{M})}(\Omega)$. Given an open subset $\Omega$ of $\mathbb{R}^{r}$, the Fréchet space $\mathcal{B}_{\left(\boldsymbol{M}_{j}\right)}(\Omega)$ is the vector space of functions $f \in \mathcal{C}^{\infty}(\Omega)$ such that

$$
\|f\|_{\Omega, j, h}:=\sup _{\alpha \in \mathbb{N}_{0}^{r}} \frac{\left\|\mathrm{D}^{\alpha} f\right\|_{\Omega}}{h^{|\alpha|} M_{j,|\alpha|}}<\infty, \quad \forall h>0,
$$

endowed with the countable system of norms $\left\{\|\cdot\|_{\Omega, j, 1 / m}: m \in \mathbb{N}\right\}$.

The Fréchet space $\mathcal{B}_{(\mathfrak{M})}(\Omega)$ is then defined as the projective limit of the sequence $\left(\mathcal{B}_{\left(\boldsymbol{M}_{j}\right)}(\Omega)\right)_{j \in \mathbb{N}}$.

The space $\mathcal{D}_{(\mathfrak{M})}(K)$. Given a compact subset $K$ of $\mathbb{R}^{r}$, the Fréchet space $\mathcal{D}_{(\mathfrak{M})}(K)$ is the topological subspace of $\mathcal{B}_{(\mathfrak{M})}\left(\mathbb{R}^{r}\right)$ consisting of the elements with support contained in $K$.

The Banach spaces $\mathcal{B}_{j, r}$ and the Fréchet spaces $\mathcal{E}_{(\mathfrak{M})}\left([-1,1]^{r}\right)$. The Banach space $\mathcal{B}_{j, r}$ is the vector space of functions $f \in \mathcal{C}^{\infty}\left([-1,1]^{r}\right)$ such that

$$
\|f\|_{j, r}:=\sup _{\alpha \in \mathbb{N}_{0}^{r}} \frac{j^{|\alpha|}\left\|\mathrm{D}^{\alpha} f\right\|_{[-1,1]^{r}}}{M_{j,|\alpha|}}<\infty,
$$

endowed with the norm $\|\cdot\|_{j, r}$.

The Fréchet space $\mathcal{E}_{(\mathfrak{M})}\left([-1,1]^{r}\right)$ is then defined as the projective limit of the sequence $\left(\mathcal{B}_{j, r}\right)_{j \in \mathbb{N}}$.

\section{Study of the space $\mathcal{E}_{(\mathfrak{M})}\left([-1,1]^{r}\right)$}

Proposition 6.1. For every $j, r \in \mathbb{N}$, there is a constant $B_{j, r}>1$ such that, for every $\alpha, \gamma \in \mathbb{N}_{0}^{r}$,

$$
\left|a_{\gamma}(f)\right| \leq 2^{r} \frac{2^{|\alpha|} B_{j, r}^{|\alpha|}}{\gamma^{\alpha}} M_{j, \alpha}\|f\|_{j+r-1, r}, \quad \forall f \in \mathcal{B}_{j+r-1, r} .
$$

In particular, for every $j, r \in \mathbb{N},\left(T_{\gamma}, a_{\gamma}(\cdot)\right)_{\gamma \in \mathbb{N}_{0}^{r}}$ is a biorthogonal system in $\mathcal{B}_{j+r-1, r}$.

Proof. For every $f \in \mathcal{B}_{j+r-1, r}$ and $\alpha, \delta \in \mathbb{N}_{0}^{r}$ such that $\delta \leq \alpha$, we clearly have

$$
\begin{aligned}
\frac{1}{\delta !}\left\|f^{(\delta)}\right\|_{[-1,1]^{r}} & \leq \frac{1}{\delta !}\|f\|_{j+r-1, r} \frac{M_{j+r-1,|\delta|}}{(j+r-1)^{|\delta|}} \\
& \leq B_{j, r}^{|\delta|} \frac{M_{j, \delta}}{\delta !}\|f\|_{j+r-1, r} \leq B_{j, r}^{|\alpha|} \frac{M_{j, \alpha}}{\alpha !}\|f\|_{j+r-1, r}
\end{aligned}
$$


for some constant $B_{j, r}>1$ independent of $f$ (if $r=1$, any number $B_{j, 1}>1$ is suitable; if $r \geq 2$, we use Proposition 4.2). So the announced inequality is an immediate consequence of the estimate $(2)$ of $\left|a_{\gamma}(f)\right|$.

The particular case is clear: it is a fact that $a_{\gamma}\left(T_{\delta}\right)$ is equal to 1 if $\gamma=\delta$ and to 0 otherwise. Moreover the established inequality implies that the linear functionals $a_{\gamma}(\cdot)$ are continuous on $\mathcal{B}_{j+r-1, r}$.

Proposition 6.2. For every $j, r \in \mathbb{N}$, the canonical injection from $\mathcal{B}_{j+r, r}$ into $\mathcal{B}_{j, r}$ is nuclear.

Proof. For every $\beta, \gamma \in \mathbb{N}_{0}^{r}$, we use the V. A. Markov inequality to estimate the derivatives of $T_{\gamma}$, and Proposition 6.1 with the special value $\alpha=\left(2 \beta_{1}+2, \ldots, 2 \beta_{r}+2\right)$ to estimate $\left|a_{\gamma}(f)\right|$. We thus get a constant $B_{j+1, r}>1$ such that

$\frac{j^{|\beta|}\left|a_{\gamma}(f)\right|\left\|\mathrm{D}^{\beta} T_{\gamma}\right\|_{[-1,1]^{r}}}{M_{j,|\beta|}} \leq \frac{j^{|\beta|} 2^{r} 2^{2|\beta|+2 r} B_{j+1, r}^{2|\beta|+2 r}}{M_{j,|\beta|}} \frac{1}{\gamma_{1}^{* 2} \cdots \gamma_{r}^{* 2}} \frac{M_{j+1, \alpha}}{\beta !}\|f\|_{j+r, r}$ for every $f \in \mathcal{B}_{j+r, r}$.

For $A=4 j B_{j+1, r}^{2}$, condition $\left(\mathfrak{m}_{4}\right)$ provides a constant $A_{j}>1$ such that

$$
M_{j+1,2(p+1)} \leq A_{j} A^{-p} p ! M_{j, p}, \quad \forall p \in \mathbb{N}_{0} .
$$

This leads to the following estimate:

$$
\frac{M_{j+1, \alpha}}{\beta !}=\frac{M_{j+1,2\left(\beta_{1}+1\right)}}{\beta_{1} !} \cdots \frac{M_{j+1,2\left(\beta_{r}+1\right)}}{\beta_{r} !} \leq A_{j}^{r} A^{-|\beta|} M_{j, \beta} \leq A_{j}^{r} A^{-|\beta|} M_{j,|\beta|} .
$$

Therefore we get

$$
\left|a_{\gamma}(f)\right|\left\|T_{\gamma}\right\|_{j, r} \leq C_{j, r} \frac{1}{\gamma_{1}^{* 2} \ldots \gamma_{r}^{* 2}}\|f\|_{j+r, r}, \quad \forall f \in \mathcal{B}_{j+r, r},
$$

with $C_{j, r}=2^{3 r} A_{j}^{r} B_{j+1, r}^{2 r}>0$ and we conclude at once since the series $\sum_{\gamma \in \mathbb{N}_{0}^{r}}\left(\gamma_{1}^{* 2} \cdots \gamma_{r}^{* 2}\right)^{-1}$ converges.

Together with inequalities (3), Propositions 6.1 and 6.2 lead to the following result.

Theorem 6.3. For every $r \in \mathbb{N}$, the family $\left(T_{\gamma}, a_{\gamma}(\cdot)\right)_{\gamma \in \mathbb{N}_{0}^{r}}$ is an absolute Schauder basis in the Fréchet nuclear space $\mathcal{E}_{(\mathfrak{M})}\left([-1,1]^{r}\right)$.

Notation. As $\mathcal{E}_{(\mathfrak{M})}([-1,1])$ is a Fréchet nuclear space, the tensor products

$$
\mathcal{E}_{(\mathfrak{M})}([-1,1]) \widehat{\otimes}_{\mathcal{E}} \cdots \widehat{\otimes}_{\mathcal{E}} \mathcal{E}_{(\mathfrak{M})}([-1,1])
$$

and

$$
\mathcal{E}_{(\mathfrak{M})}([-1,1]) \widehat{\otimes}_{\pi} \cdot \stackrel{r}{\widehat{\otimes}_{\pi}} \mathcal{E}_{(\mathfrak{M})}([-1,1])
$$

coincide. To shorten notation, let us designate them by $\widehat{\otimes}^{r} \mathcal{E}_{(\mathfrak{M})}([-1,1])$. 
Proposition 6.4. For every integer $r \geq 2$, the Fréchet nuclear spaces $\widehat{\bigotimes}^{r} \mathcal{E}_{(\mathfrak{M})}([-1,1])$ and $\mathcal{E}_{(\mathfrak{M})}\left([-1,1]^{r}\right)$ coincide.

Proof. Let $\mathcal{E}$ denote the tensor product

$$
\mathcal{E}_{(\mathfrak{M})}([-1,1]) \otimes \stackrel{r}{ } \cdot \mathcal{E}_{(\mathfrak{M})}([-1,1]) .
$$

By Theorem 6.3 , we already know that $\mathcal{E}$ is a dense vector subspace of $\mathcal{E}_{(\mathfrak{M})}\left([-1,1]^{r}\right)$.

We first prove that on $\mathcal{E}$ the $\pi$-topology is finer than the topology induced by $\mathcal{E}_{(\mathfrak{M})}\left([-1,1]^{r}\right)$. For every $j \in \mathbb{N}$, it is well known that the set $U_{j}$ of linear combinations

$$
\sum_{s=1}^{l} b_{s} f_{s, 1}\left(x_{1}\right) \cdots f_{s, r}\left(x_{r}\right)=\sum_{s=1}^{l} b_{s} f_{s, 1} \otimes \cdots \otimes f_{s, r}(x)
$$

with $l \in \mathbb{N}, b_{1}, \ldots, b_{l} \in \mathbb{K}$ such that $\sum_{s=1}^{l}\left|b_{s}\right| \leq 1$, and $f_{s, k} \in \mathcal{E}_{(\mathfrak{M})}([-1,1])$ such that $\left\|f_{s, k}\right\|_{j, 1} \leq 1$ for all $s \in\{1, \ldots, l\}$ and $k \in\{1, \ldots, r\}$, is a neighbourhood in $\mathcal{E}$ endowed with the $\pi$-topology. To conclude it is then enough to verify that, for every $j \in \mathbb{N}, U_{j}$ is a subset of the closed unit semi-ball of $\mathcal{E}_{(\mathfrak{M})}\left([-1,1]^{r}\right)$ for the semi-norm $\|\cdot\|_{j, r}$. This is straightforward: for every element $f=\sum_{s=1}^{l} b_{s} f_{s, 1} \otimes \cdots \otimes f_{s, r}$ of $U_{j}$, one just has to note that, for every $s \in\{1, \ldots, l\}$,

$$
\left\|f_{s, 1} \otimes \cdots \otimes f_{s, r}\right\|_{j, r} \leq\left\|f_{s, 1}\right\|_{j, 1} \cdots\left\|f_{s, r}\right\|_{j, 1} \leq 1 .
$$

Now we prove that on $\mathcal{E}$, the $\varepsilon$-topology is weaker than the topology induced by $\mathcal{E}_{(\mathfrak{M})}\left([-1,1]^{r}\right)$. For any elements $u_{1}, \ldots, u_{r}$ of the topological dual of $\mathcal{E}_{(\mathfrak{M})}([-1,1])$ and $f \in \mathcal{E}_{(\mathfrak{M})}\left([-1,1]^{r}\right)$, set

$$
\left\langle f, u_{1} \otimes \cdots \otimes u_{r}\right\rangle:=\sum_{\gamma \in \mathbb{N}_{0}^{r}} a_{\gamma}(f)\left\langle u_{1}, T_{\gamma_{1}}\right\rangle \cdots\left\langle u_{r}, T_{\gamma_{r}}\right\rangle .
$$

For every $j \in \mathbb{N}$, let moreover $V_{j}$ denote the polar set of

$$
\left\{f \in \mathcal{E}_{(\mathfrak{M})}([-1,1]):\|f\|_{[-1,1], j, 1 / j} \leq 1\right\}
$$

in the topological dual of $\mathcal{E}_{(\mathfrak{M})}([-1,1])$. If $u_{1}, \ldots, u_{r} \in V_{j}$, we get

with

$$
\left|\left\langle f, u_{1} \otimes \cdots \otimes u_{r}\right\rangle\right| \leq \sum_{\gamma \in \mathbb{N}_{0}^{r}}\left|a_{\gamma}(f)\right|\left\|T_{\gamma_{1}}\right\|_{j, 1} \cdots\left\|T_{\gamma_{r}}\right\|_{j, 1}
$$

$$
\left\|T_{\gamma_{1}}\right\|_{j, 1} \cdots\left\|T_{\gamma_{r}}\right\|_{j, 1}=\sup _{\alpha \in \mathbb{N}_{0}^{r}} \frac{j^{|\alpha|}\left\|\mathrm{D}^{\alpha} T_{\gamma}\right\|_{[-1,1]^{r}}}{M_{j, \alpha}} \leq \sup _{\alpha \in \mathbb{N}_{0}^{r}} \frac{B_{j, r}^{|\alpha|} j^{|\alpha|}\left\|\mathrm{D}^{\alpha} T_{\gamma}\right\|_{[-1,1]^{r}}}{M_{j+r-1,|\alpha|}}
$$

for some $B_{j, r}>1$ independent of $\gamma$ by Proposition 4.2. So if $m_{j, r}$ is an integer such that $m_{j, r}>j+r-1$ and $m_{j, r}>j B_{j, r}$, we have

$$
\left\|T_{\gamma_{1}}\right\|_{j, 1} \cdots\left\|T_{\gamma_{r}}\right\|_{j, 1} \leq\left\|T_{\gamma}\right\|_{m_{j, r}, r}
$$


for every $\gamma \in \mathbb{N}_{0}^{r}$. Now we apply inequality (3) to get

$$
\left|\left\langle f, u_{1} \otimes \cdots \otimes u_{r}\right\rangle\right| \leq C_{m_{j, r}, r}\|f\|_{m_{j, r}, r} \sum_{\gamma \in \mathbb{N}_{0}^{r}} \frac{1}{\gamma_{1}^{* 2} \cdots \gamma_{r}^{* 2}}
$$

for every $f \in \mathcal{E}_{(\mathfrak{M})}\left([-1,1]^{r}\right)$, for some constant $C_{m_{j, r}, r}>0$ independent of $f$. Therefore $u_{1} \otimes \cdots \otimes u_{r}$ is a continuous linear functional on $\mathcal{E}_{(\mathfrak{M})}\left([-1,1]^{r}\right)$ and

$$
\left\{u_{1} \otimes \cdots \otimes u_{r}: u_{1}, \ldots, u_{r} \in V_{j}\right\}
$$

is an equicontinuous subset of the topological dual of $\mathcal{E}_{(\mathfrak{M})}\left([-1,1]^{r}\right)$.

Hence the conclusion follows, since the topologies $\varepsilon$ and $\pi$ coincide on $\mathcal{E}$.

7. Non-extension result. It is clear that the restriction map

$$
R: \mathcal{B}_{(\mathfrak{M})}\left(\mathbb{R}^{r}\right) \rightarrow \Lambda_{(\mathfrak{M})}^{(r)}, \quad f \mapsto\left(\mathrm{D}^{\gamma} f(0)\right)_{\gamma \in \mathbb{N}_{0}^{r}},
$$

is well defined, linear and continuous.

Proposition 7.1. For every $r \in \mathbb{N}$, there is no continuous linear extension map from $\Lambda_{(\mathfrak{M})}^{(r)}$ into $\mathcal{B}_{(\mathfrak{M})}\left(\mathbb{R}^{r}\right)$.

Proof. We proceed by induction on $r$.

CASE $r=1$. Suppose that there is a continous linear extension map $T$ from $\Lambda_{(\mathfrak{M})}^{(1)}$ into $\mathcal{B}_{(\mathfrak{M})}(\mathbb{R})$. Then there are $\left.\left.r, s \in \mathbb{N}, h, k \in\right] 0,1\right]$ and $H, K>1$ such that

$$
\|T \boldsymbol{a}\|_{\mathbb{R}, 1,1} \leq H|\boldsymbol{a}|_{s, h} \quad \text { and } \quad\|T \boldsymbol{a}\|_{\mathbb{R}, s+1,1} \leq K|\boldsymbol{a}|_{r, k}
$$

for every $\boldsymbol{a} \in \Lambda_{(\mathfrak{M})}^{(1)}$. For every $p \in \mathbb{N}_{0}$, denote by $e_{p}$ the $p$ th unit vector of $\Lambda_{(\mathfrak{M})}^{(1)}$ and set $\chi_{p}:=T e_{p}$. We then have

$$
\left\|\chi_{p}\right\|_{\mathbb{R}, 1,1} \leq \frac{H}{h^{p} M_{s, p}} \quad \text { and } \quad\left\|\chi_{p}\right\|_{\mathbb{R}, s+1,1} \leq \frac{K}{k^{p} M_{r, p}} .
$$

Moreover for every $x>0$, the Taylor development provides some $y \in] 0, x]$ such that

$$
\chi_{p}^{(p)}(x)=\chi_{p}^{(p)}(0)+\frac{x^{p}}{p !} \chi_{p}^{(2 p)}(y)=1+\frac{x^{p}}{p !} \chi_{p}^{(2 p)}(y)
$$

hence

$$
\left|\chi_{p}^{(p)}(x)-1\right| \leq \frac{x^{p}}{p !}\left\|\chi_{p}\right\|_{\mathbb{R}, s+1,1} M_{s+1,2 p} \leq \frac{x^{p}}{p !} \frac{K M_{s+1,2 p}}{k^{p} M_{r, p}} .
$$

So if we define $\tau_{p}>0$ by $\tau_{p}^{p}=p ! k^{p} M_{r, p} /\left(2 K M_{s+1,2 p}\right)$, we get

$$
\left.\left.\left|\chi_{p}^{(p)}(x)-1\right| \leq \frac{\tau_{p}^{p}}{p !} \frac{K M_{s+1,2 p}}{k^{p} M_{r, p}}=\frac{1}{2}, \quad \forall x \in\right] 0, \tau_{p}\right],
$$


hence $\Re \chi_{p}^{(p)}(x) \geq 1 / 2$ and therefore $\Re \chi_{p}(x) \geq x^{p} /(2 p$ !) for every $\left.x \in] 0, \tau_{p}\right]$. This leads to

$$
\frac{k^{p} M_{r, p}}{4 K M_{s+1,2 p}}=\frac{\tau_{p}^{p}}{2 p !} \leq \Re \chi_{p}\left(\tau_{p}\right) \leq\left\|\chi_{p}\right\|_{\mathbb{R}, 1,1} M_{1,0} \leq \frac{H}{h^{p} M_{s, p}} .
$$

Now we note that condition $\left(\mathfrak{m}_{4}\right)$ applied with $A=1$ provides a constant $B_{s}>0$ such that $M_{s+1,2(p+1)} \leq B_{s} p ! M_{s, p}$ for every $p \in \mathbb{N}$. So the preceding inequalities lead to

$$
\frac{M_{r, p}}{p !} \leq \frac{4 H K M_{s+1,2 p}}{p ! h^{p} k^{p} M_{s, p}} \leq \frac{4 H K}{p ! h^{p} k^{p}} \frac{M_{s+1,2(p+1)}}{M_{s, p}} \leq \frac{4 H K}{h^{p} k^{p}} B_{s}
$$

and we obtain the existence of some $B>0$ such that $M_{r, p} / p ! \leq B^{p+1}$ for every $p \in \mathbb{N}_{0}$. As by Proposition 4.1 , there is $c>0$ such that $M_{r, p} / p$ ! $\geq$ $c(2 B)^{p+1}$ for every $p \in \mathbb{N}_{0}$, we arrive at the contradiction $c(2 B)^{p+1} \leq B^{p+1}$ for every $p \in \mathbb{N}_{0}$.

General case. Suppose that for some $r>1$, there is a continuous linear extension map $S$ from $\Lambda_{(\mathfrak{M})}^{(r)}$ into $\mathcal{B}_{(\mathfrak{M})}\left(\mathbb{R}^{r}\right)$. Of course, the map $V: \Lambda_{(\mathfrak{M})}^{(1)}$ $\rightarrow \Lambda_{(\mathfrak{M})}^{(r)}$ defined by

$$
(V \boldsymbol{a})_{\gamma}= \begin{cases}0 & \text { if }\left(\gamma_{2}, \ldots, \gamma_{r}\right) \neq 0 \\ \left(a_{\gamma_{1}}, 0, \ldots, 0\right) & \text { otherwise }\end{cases}
$$

is well defined, linear and continuous. Moreover the map $R_{1}$ from $\mathcal{B}_{(\mathfrak{M})}\left(\mathbb{R}^{r}\right)$ into $\mathcal{B}_{(\mathfrak{M})}(\mathbb{R})$ defined by $\left(R_{1} f\right)(t)=f(t, 0, \ldots, 0)$ for every $t \in \mathbb{R}$ is also well defined, linear and continuous. Therefore the map $R_{1} \circ S \circ V$ is continuous and linear from $\Lambda_{(\mathfrak{M})}^{(1)}$ into $\mathcal{B}_{(\mathfrak{M})}(\mathbb{R})$. As it is clearly an extension map, we have arrived at a contradiction.

\section{Conditions $\left(\mathfrak{m}_{5}\right)$ and $\left(\mathfrak{m}_{5}^{\prime}\right)$}

Definition. (a) The matrix $\mathfrak{m}$ satisfies condition $\left(\mathfrak{m}_{5}\right)$ if there is some $\alpha>1$ such that $\sum_{p=1}^{\infty} p^{\alpha} / m_{j, p}<\infty$ for every $j \in \mathbb{N}$.

(b) The matrix $\mathfrak{m}$ satisfies condition $\left(\mathfrak{m}_{5}^{\prime}\right)$ if there is some $\beta>2$ such that $\lim _{p \rightarrow \infty} p !^{\beta} / M_{j, p}=0$ for every $j \in \mathbb{N}$.

Proposition 8.1. The matrix $\mathfrak{m}$ satisfies condition $\left(\mathfrak{m}_{5}\right)$ if and only if it satisfies condition $\left(\mathfrak{m}_{5}^{\prime}\right)$.

Proof. We will prove that if $\mathfrak{m}$ satisfies $\left(\mathfrak{m}_{5}\right)$ with $\alpha>1$, then it satisfies $\left(\mathfrak{m}_{5}^{\prime}\right)$ with $\beta=1+\alpha$. For every $j \in \mathbb{N}$, the sequence $\left(m_{j, p}^{\prime}\right)_{p \in \mathbb{N}_{0}}$ defined by $m_{j, 0}^{\prime}=1$ and $m_{j, p}^{\prime}=p^{-\alpha} m_{j, p}$ for every $p \in \mathbb{N}$ is non-quasianalytic. Therefore the Denjoy-Carleman theorem (cf. [3, Theorem 1.3.8]) gives $\sum_{p=1}^{\infty} 1 / L_{j, p}<\infty$ if we set

$$
L_{j, p}=\inf \left\{\left(M_{j, k}^{\prime}\right)^{1 / k}: k \geq p\right\}, \quad \forall p \in \mathbb{N} .
$$


As $\left(1 / L_{j, p}\right)_{p \in \mathbb{N}}$ is a decreasing sequence of positive numbers, this leads to $p / L_{j, p} \rightarrow 0$. The conclusion is then clear since

$$
\frac{p !^{1+\alpha}}{M_{j, p}} \leq \frac{p^{p} p^{\alpha}}{M_{j, p}}=\frac{p^{p}}{M_{j, p}^{\prime}} \leq\left(\frac{p}{L_{j, p}}\right)^{p}, \quad \forall p \in \mathbb{N} .
$$

If $\mathfrak{m}$ satisfies $\left(\mathfrak{m}_{5}^{\prime}\right)$ with $\beta>2$, the following argument proves that it satisfies $\left(\mathfrak{m}_{5}\right)$ for any $\left.\alpha \in\right] 1, \beta-1[$. Let $j \in \mathbb{N}$ and choose $\gamma \in] 2, \beta[$. An immediate application of the Stirling formula gives $\lim _{p \rightarrow \infty} p^{\gamma p} /(p !)^{\beta}=0$, hence $\lim _{p \rightarrow \infty} p^{\gamma p} / M_{j, p}=0$. So there is $p_{j} \in \mathbb{N}$ such that $p^{\gamma p}<M_{j, p}$, hence $p^{\gamma}<m_{j, p}$ for every $p \geq p_{j}$. Choosing now $\alpha>1$ such that $\gamma-\alpha>1$, we get

$$
\frac{p^{\alpha}}{m_{j, p}}=\frac{p^{\gamma}}{m_{j, p}} \frac{1}{p^{\gamma-\alpha}}<\frac{1}{p^{\gamma-\alpha}}, \quad \forall p \geq p_{j},
$$

hence $\sum_{p=1}^{\infty} p^{\alpha} / m_{j, p}<\infty$.

\section{Extension results}

Construction. If the matrix $\mathfrak{m}$ satisfies condition $\left(\mathfrak{m}_{5}\right)$ with $\alpha>1$, we make the following choices.

a) The integers $p_{j}$ and the numbers $n_{p}$ and $N_{p}$. We first choose a strictly increasing sequence $\left(p_{j}\right)_{j \in \mathbb{N}}$ of positive integers such that

$$
\sum_{p=p_{j}+1}^{\infty} \frac{p^{\alpha}}{m_{j+1, p}}<2^{-j}, \quad \forall j \in \mathbb{N} .
$$

We then define the sequence $\left(n_{p}\right)_{p \in \mathbb{N}_{0}}$ as follows:

$$
n_{p}= \begin{cases}1 & \text { if } p=0, \\ p^{-\alpha} m_{1, p} & \text { if } p \in\left\{1, \ldots, p_{1}\right\}, \\ p^{-\alpha} m_{j+1, p} & \text { if } p \in\left\{p_{j}+1, \ldots, p_{j+1}\right\} \text { and } j \in \mathbb{N} .\end{cases}
$$

Of course we then set $N_{p}=n_{0} \cdots n_{p}$ for every $p \in \mathbb{N}_{0}$.

Note that, for every $j \in \mathbb{N}$, there is clearly a constant $c_{j}>1$ such that $n_{p} \leq c_{j} p^{-\alpha} m_{j, p}$ for every $p \in \mathbb{N}_{0}$, hence

$$
N_{p} \leq c_{j}^{p} p !^{-\alpha} M_{j, p}, \quad \forall p \in \mathbb{N}_{0} .
$$

b) The functions $S_{n}$. For $a:=\sum_{p=1}^{\infty} 1 / n_{p}$, a slight enhancement of property 1.3.5 of [3] provides the existence of a function $\varphi \in \mathcal{D}([-a, a])$ such that $0 \leq \varphi \leq 1$ as well as $\varphi^{(p)}(0)=\delta_{p, 0}$ and $\left\|\varphi^{(p)}\right\|_{\mathbb{R}} \leq 2^{p} N_{p}$ for every $p \in \mathbb{N}_{0}$. 
For every $n \in \mathbb{N}_{0}$, we then set $v_{n}(x)=\varphi\left(a\left(1+n^{2}\right) x\right)$ for every $x \in \mathbb{R}$ and define the function $u_{n}$ on $\mathbb{R}$ by setting

$$
u_{n}(x):= \begin{cases}0 & \text { if } x<-1-1 /\left(1+n^{2}\right), \\ v_{n}(x+1) & \text { if }-1-1 /\left(1+n^{2}\right) \leq x \leq-1, \\ 1 & \text { if }-1<x<1 \\ v_{n}(x-1) & \text { if } 1 \leq x \leq 1+1 /\left(1+n^{2}\right), \\ 0 & \text { if } 1+1 /\left(1+n^{2}\right)<x .\end{cases}
$$

Finally, for every $n \in \mathbb{N}_{0}$, we set $S_{n}:=T_{n} u_{n}$. It is a direct matter to verify that $S_{n}$ belongs to $\mathcal{C}^{\infty}(\mathbb{R})$, is an extension of $\left.T_{n}\right|_{[-1,1]}$ and has its support contained in $\left[-1-1 /\left(1+n^{2}\right), 1+1 /\left(1+n^{2}\right)\right]$.

c) The functions $S_{\gamma}$. Now everything is set up to introduce the following functions: for every $\gamma \in \mathbb{N}_{0}^{r}$, we define the function $S_{\gamma}$ on $\mathbb{R}^{r}$ by

$$
S_{\gamma}(x)=S_{\gamma_{1}}\left(x_{1}\right) \cdots S_{\gamma_{r}}\left(x_{r}\right), \quad \forall x \in \mathbb{R}^{r} .
$$

It is clear that $S_{\gamma}$ belongs to $\mathcal{C}^{\infty}\left(\mathbb{R}^{r}\right)$, is an extension of $\left.T_{\gamma}\right|_{[-1,1]^{r}}$ and has its support contained in $[-2,2]^{r}$. A lot more can be said.

Lemma 9.1. If the matrix $\mathfrak{m}$ satisfies condition $\left(\mathfrak{m}_{5}\right)$, then there is a constant $b>1$ such that $\left\|S_{0}^{(p)}\right\|_{\mathbb{R}} \leq b^{p+1} N_{p}$ and $\left\|S_{n}^{(p)}\right\|_{\mathbb{R}} \leq\left(b n^{2}\right)^{p+1} N_{p}$ for every $n \in \mathbb{N}$ and $p \in \mathbb{N}_{0}$.

Proof. For every $n \in \mathbb{N}, x \in \mathbb{R}$ such that $|x| \leq 1+1 /\left(1+n^{2}\right)$ and $p \in \mathbb{N}_{0}$, Proposition 3.1 and the preceding construction lead to

$$
\begin{aligned}
\left|S_{n}^{(p)}(x)\right| & \leq \sum_{l=0}^{p}\left(\begin{array}{l}
p \\
l
\end{array}\right)\left|T_{n}^{(l)}(x)\right|\left|u_{n}^{(p-l)}(x)\right| \\
& \leq \sum_{l=0}^{p}\left(\begin{array}{l}
p \\
l
\end{array}\right) e \frac{n^{2 l}}{l !} 2^{p-l} N_{p-l} a^{p-l}\left(1+n^{2}\right)^{p-l} .
\end{aligned}
$$

As the series $\sum_{p=0}^{\infty} 1 / n_{p}$ converges, we have $n_{p} \geq 1$ for $p$ large; this implies the existence of a constant $c>1$ such that $N_{q} \leq c N_{p}$ for every $p, q \in \mathbb{N}$ such that $q \leq p$. Therefore we get

$$
\begin{aligned}
\left\|S_{n}^{(p)}\right\|_{\mathbb{R}} & \leq c e N_{p} \sum_{l=0}^{p}\left(\begin{array}{l}
p \\
l
\end{array}\right) n^{2 l}\left(2 a\left(1+n^{2}\right)\right)^{p-l} \\
& \leq \operatorname{ceN} N_{p}\left(n^{2}+2 a\left(1+n^{2}\right)\right)^{p}, \quad \forall n \in \mathbb{N}, p \in \mathbb{N}_{0} .
\end{aligned}
$$

As it is clear that we also have

$$
\left\|S_{0}^{(p)}\right\|_{\mathbb{R}}=\left\|u_{0}^{(p)}\right\|_{\mathbb{R}} \leq(2 a)^{p} N_{p}, \quad \forall p \in \mathbb{N}_{0}
$$

we conclude at once. 
Proposition 9.2. If the matrix $\mathfrak{m}$ satisfies condition $\left(\mathfrak{m}_{5}\right)$, then there is $q \in \mathbb{N}$ such that, for every $j, r \in \mathbb{N}$, there is a constant $D_{j, r, q}>0$ such that

$$
\left\|S_{\gamma}\right\|_{\mathbb{R}^{r}, j, 1 / j} \leq D_{j, r, q}\left\|T_{\gamma}\right\|_{j+q+2 r, r}, \quad \forall \gamma \in \mathbb{N}_{0}^{r} .
$$

In particular, for every $\gamma \in \mathbb{N}_{0}^{r}$, the extension $S_{\gamma}$ of $\left.T_{\gamma}\right|_{[-1,1]^{r}}$ belongs to $\mathcal{D}_{(\mathfrak{M})}\left([-2,2]^{r}\right)$.

Proof. Let us first remark that, for every $j \in \mathbb{N}$, condition $\left(\mathfrak{m}_{4}\right)$ applied with $A=1$ provides a constant $E_{j}>1$ such that

$$
M_{j+1,2(p+1)} \leq E_{j} p ! M_{j, p}, \quad \forall p \in \mathbb{N}_{0},
$$

hence

$$
M_{j+1, p}^{2} \leq M_{j+1,2(p+1)} \leq E_{j} p ! M_{j, p}, \quad \forall p \in \mathbb{N}_{0}
$$

Now we fix $q$ by the following observation: as the matrix $\mathfrak{m}$ satisfies $\left(\mathfrak{m}_{5}\right)$, there is $\lambda>1$ such that $\sum_{p=1}^{\infty} p^{\lambda} / m_{j, p}<\infty$ for every $j \in \mathbb{N}$, so we can choose $q \in \mathbb{N}$ with $2^{-q}<\lambda-1$.

Next, for every $j, r \in \mathbb{N}$, we proceed as follows. First of all, to shorten notation, we set $\left\|T_{\gamma}\right\|_{*}=\left\|T_{\gamma}\right\|_{j+q+2 r, r}$ for every $\gamma \in \mathbb{N}_{0}^{r}$. Then we recall that Proposition 6.1 provides some $B=B_{j+q+r+1, r}>1$ depending only on $j, r$ and $q$ such that

$$
1=\left|a_{\gamma}\left(T_{\gamma}\right)\right| \leq 2^{r} \frac{(2 B)^{|\beta|}}{\gamma^{\beta}} M_{j+q+r+1, \beta}\left\|T_{\gamma}\right\|_{*}, \quad \forall \beta, \gamma \in \mathbb{N}_{0}^{r} .
$$

So, for every $\alpha \in \mathbb{N}_{0}^{r}$, using successively Lemma 9.1, inequality (4) and (7) with $\beta=\left(2\left(\alpha_{1}+1\right), \ldots, 2\left(\alpha_{r}+1\right)\right.$ and setting $A=8 b c_{j+q+r} B^{2}$, we obtain

$$
\left\|S_{\gamma}^{(\alpha)}\right\|_{\mathbb{R}^{r}}=\prod_{k=1}^{r}\left\|S_{\gamma_{k}}^{\left(\alpha_{k}\right)}\right\|_{\mathbb{R}} \leq A^{r}\left\|T_{\gamma}\right\|_{*} \prod_{k=1}^{r} \frac{A^{\alpha_{k}}}{\alpha_{k} !^{\lambda}} M_{j+q+r, \alpha_{k}} M_{j+q+r+1,2\left(\alpha_{k}+1\right)} .
$$

Now for every $k \in\{1, \ldots, r\}$, we use (5) and (6) to get

$$
M_{j+q+r, \alpha_{k}} M_{j+q+r+1,2\left(\alpha_{k}+1\right)} \leq \alpha_{k} !^{2} E_{j+q+r} E_{j+q+r-1} M_{j+q+r-1, \alpha_{k}}
$$

and then we note that a repeated use of inequalities of type (6) leads to

$$
\begin{aligned}
E_{j+q+r-1} \frac{M_{j+q+r-1, \alpha_{k}}}{\alpha_{k} !} & \leq E_{j+q+r-1} E_{j+q+r-2}^{2^{-1}}\left(\frac{M_{j+q+r-2, \alpha_{k}}}{\alpha_{k} !}\right)^{2^{-1}} \leq \cdots \\
& \leq E_{j+q+r-1} E_{j+q+r-2}^{2^{-1}} \cdots E_{j+r-1}^{2^{-q}}\left(\frac{M_{j+r-1, \alpha_{k}}}{\alpha_{k} !}\right)^{2^{-q}} .
\end{aligned}
$$

Setting $E=E_{j+q+r} \prod_{k=0}^{q} E_{j+q+r-1-k}^{2^{-k}}$ and putting all this information together yields

$$
\left\|S_{\gamma}^{(\alpha)}\right\|_{\mathbb{R}^{r}} \leq(A E)^{r}\left\|T_{\gamma}\right\|_{*} \prod_{k=1}^{r} \frac{A^{\alpha_{k}}}{\alpha_{k} !^{\lambda-3}}\left(\frac{M_{j+r-1, \alpha_{k}}}{\alpha_{k} !}\right)^{2^{-q}}
$$


hence

$$
\begin{aligned}
\frac{j^{|\alpha|}\left\|S_{\gamma}^{(\alpha)}\right\|_{\mathbb{R}^{r}}}{M_{j,|\alpha|}} & \leq\left\|S_{\gamma}^{(\alpha)}\right\|_{\mathbb{R}^{r}} \prod_{k=1}^{r} \frac{j^{\alpha_{k}}}{M_{j, \alpha_{k}}} \leq\left\|S_{\gamma}^{(\alpha)}\right\|_{\mathbb{R}^{r}} \prod_{k=1}^{r} \frac{j^{\alpha_{k}}}{M_{j+r-1, \alpha_{k}}} \\
& \leq(A E)^{r}\left\|T_{\gamma}\right\|_{*} \prod_{k=1}^{r} \frac{(j A)^{\alpha_{k}}}{\alpha_{k} !^{\lambda-1-2^{q}}}\left(\frac{\alpha_{k} !^{2}}{M_{j+r-1, \alpha_{k}}}\right)^{1-2^{-q}}
\end{aligned}
$$

Finally, on the one hand the sequence $\left(p / m_{j, p}\right)_{p \in \mathbb{N}}$ of positive numbers is decreasing and satisfies $\sum_{p=1}^{\infty} p / m_{j, p}<\infty$; this implies $\lim p^{2} / m_{j, p}=0$, hence $\lim p !^{2} / M_{j, p}=0$. On the other hand, the sequence $\left((j A)^{p} / p !^{\lambda-1-2^{-q}}\right)_{p \in \mathbb{N}}$ converges to 0 . These two facts and the last inequality together provide the existence of a constant $D_{j, r, q}>0$ such that

$$
\frac{j^{|\alpha|}\left\|S_{\gamma}^{(\alpha)}\right\| \mathbb{R}^{r}}{M_{j,|\alpha|}} \leq D_{j, r, q}\left\|T_{\gamma}\right\|_{*}, \quad \forall \alpha \in \mathbb{N}_{0}^{r}
$$

hence the conclusion.

THEOREM 9.3. If the matrix $\mathfrak{m}$ satisfies condition $\left(\mathfrak{m}_{5}\right)$, then for every $r \in \mathbb{N}$

is a continuous linear extension map.

$$
T: \mathcal{E}_{(\mathfrak{M})}\left([-1,1]^{r}\right) \rightarrow \mathcal{D}_{(\mathfrak{M})}\left([-2,2]^{r}\right), \quad f \mapsto \sum_{\gamma \in \mathbb{N}_{0}^{r}} a_{\gamma}(f) S_{\gamma}
$$

Proof. This is a direct consequence of Theorem 6.3 and Proposition 9.2.

\section{Application}

Proposition 10.1. If $\Phi:[0, \infty[\rightarrow[0, \infty[$ is an increasing and convex function such that $\Phi(0)=0$ and $\lim _{t \rightarrow \infty} \Phi(t) / t=\infty$, then the matrix $\mathfrak{m}=$ $\left(m_{j, p}\right)_{j \in \mathbb{N}, p \in \mathbb{N}_{0}}$ defined by $m_{j, 0}=1$ and

$$
m_{j, p}=p e^{\Phi\left(p / 8^{j}\right)-\Phi\left((p-1) / 8^{j}\right)}
$$

for every $j, p \in \mathbb{N}$ satisfies conditions $\left(\mathfrak{m}_{1}\right)$ to $\left(\mathfrak{m}_{4}\right)$.

If moreover $\Phi$ satisfies $\lim _{p \rightarrow \infty} \Phi(t) /(t \log (t))=\infty$, then $\mathfrak{m}$ also satisfies $\left(\mathfrak{m}_{5}\right)$.

Proof. By Proposition 7.1 of [8], we know that $\mathfrak{m}$ satisfies $\left(\mathfrak{m}_{1}\right)$ to $\left(\mathfrak{m}_{3}\right)$.

Let us prove that it also satisfies $\left(\mathfrak{m}_{4}\right)$. For every $p \in \mathbb{N}$, as we have $\left(\begin{array}{c}2 p+2 \\ p\end{array}\right) \leq 2^{2 p+2}, p+1 \leq 2^{p}$ and $p+2 \leq 2^{p+1}$, we get $(2 p+2) ! \leq 2^{3+4 p} p !^{2}$. So for every $j, p \in \mathbb{N}$, we have

$$
M_{j+1,2(p+1)}=(2 p+2) ! e^{\Phi\left(2(p+1) / 8^{j+1}\right)} \leq 2^{3+4 p} p !^{2} e^{\Phi\left(p /\left(2 \cdot 8^{j}\right)\right)}
$$

since $\Phi$ is an increasing function. As also $2 \Phi(t) \leq \Phi(2 t)$ for every $t \geq 0$, this 
leads to

$$
M_{j+1,2(p+1)} \leq 2^{7 p} p !^{2} e^{\Phi\left(p / 8^{j}\right)} e^{-\Phi\left(p /\left(2 \cdot 8^{j}\right)\right)}=2^{7 p} p ! M_{j, p} e^{-\phi\left(p /\left(2 \cdot 8^{j}\right)\right)}
$$

for every $j, p \in \mathbb{N}$. Now the condition $\lim \Phi(t) / t=\infty$ implies for every $A>0$ the existence of some $p_{A} \in \mathbb{N}$ such that $\Phi\left(p /\left(2 \cdot 8^{j}\right)\right) \geq$ $p\left(\log (A)+\log \left(2^{7}\right)\right)$, hence $e^{-\Phi\left(p /\left(2 \cdot 8^{j}\right)\right)} \leq A^{-p} 2^{-7 p}$ for every $p \geq p_{A}$. So altogether we have

$$
M_{j+1,2(p+1)} \leq A^{-p} p ! M_{j, p}, \quad \forall p \geq p_{A} ;
$$

hence the conclusion.

Finally, we prove that if $\Phi$ satisfies $\lim \Phi(t) /(t \log (t))=\infty$, then $\mathfrak{m}$ satisfies $\left(\mathfrak{m}_{5}^{\prime}\right)$ with $\beta=3$. Indeed, for every $j \in \mathbb{N}$, the supplementary condition provides $\lim \Phi\left(8^{-j} p\right) /(p \log (p))=\infty$, hence there is $p_{j} \in \mathbb{N}$ such that $\Phi\left(8^{-j} p\right) \geq 3 p \log (p)$ for every $p \geq p_{j}$. So for every $p \geq p_{j}$ we certainly have

$$
\frac{p !^{3}}{M_{j, p}}=\frac{p !^{2}}{e^{\Phi\left(p / 8^{j}\right)}} \leq p^{2 p} e^{-\Phi\left(p / 8^{j}\right)} \leq e^{-p \log (p)}
$$

and we conclude at once.

Let $\Phi$ be a real, increasing and convex function on $[0, \infty[$ such that $\Phi(0)=0$ and $\lim _{t \rightarrow \infty} \Phi(t) / t=\infty$.

The notations have been modified several times: the spaces $\mathrm{I}_{4, \Phi}(\{0\})$, $\mathrm{I}_{4, \Phi}([-1,1])$ and $\mathrm{I}_{4, \Phi}(\mathbb{R})$ introduced in $[1]$ become $\mathrm{I}_{\Phi}(\{0\}), \mathrm{I}_{\Phi}([-1,1])$ and $\mathrm{I}_{\Phi}(\mathbb{R})$ in $[2]$. If one considers the matrix $\mathfrak{m}$ associated to $\Phi$ in Proposition 10.1 , they coincide respectively with the spaces $\widehat{\Lambda}_{(\mathfrak{M})}, \widehat{\mathcal{E}}_{(\mathfrak{M})}([-1,1])$ and $\widehat{\mathcal{B}}_{(\mathfrak{M})}(\mathbb{R})$ of $[8]$ and with the spaces $\Lambda_{(\mathfrak{M})}^{(1)}, \mathcal{E}_{(\mathfrak{M})}([-1,1])$ and $\mathcal{B}_{(\mathfrak{M})}(\mathbb{R})$ in this paper.

Let us also recall that in [8], it is proven that there are spaces $\mathcal{B}_{(\mathfrak{M})}(\mathbb{R})$ for which there is no function $\Phi$ such that $\mathcal{B}_{(\mathfrak{M})}(\mathbb{R})=\mathrm{I}_{\Phi}(\mathbb{R})$. So Proposition 7.1 and Theorems 6.3 and 9.3 provide enhancements of the following results of Beaugendre.

THEOREM 10.2. Let $\Phi$ be a real, increasing and convex function on the interval $\left[0, \infty\left[\right.\right.$ such that $\Phi(0)=0$ and $\lim _{t \rightarrow \infty} \Phi(t) / t=\infty$.

(a) ([1, Proposition 3.1.1]) There is no continuous linear extension map from $\Lambda_{(\mathfrak{M})}^{(1)}$ into $\mathcal{B}_{(\mathfrak{M})}(\mathbb{R})$.

(b) ([2, Theorem 3.6]) The family $\left(T_{n}, a_{n}(\cdot)\right)_{n \in \mathbb{N}_{0}}$ is an absolute Schauder basis in $\mathcal{E}_{(\mathfrak{M})}([-1,1])$.

(c) $\left(\left[2\right.\right.$, Theorem 4.3]) If $\Phi$ also satisfies $\lim _{t \rightarrow \infty} \Phi(t) /(t \log (t))=\infty$, then there is a continuous linear extension map from $\mathcal{E}_{(\mathfrak{M})}([-1,1])$ into $\mathcal{B}_{(\mathfrak{M})}(\mathbb{R})$ by means of a modification of the Chebyshev polynomials. 


\section{References}

[1] P. Beaugendre, Intersection de classes non quasi-analytiques, Thèse de doctorat en sciences, Univ. de Paris XI, UFR Scientifique d'Orsay, 2404 (2002), 84 pp.

[2] - Opérateurs d'extension linéaires explicites dans des intersections de classes ultradifférentiables, Prépublication 2003-67, Univ. de Paris-Sud, Math., 35 pp.

[3] L. Hörmander, The Analysis of Linear Partial Differential Operators, Springer, 1983.

[4] B. S. Mityagin, Approximate dimension and bases in nuclear spaces, Russian Math. Surveys 16 (1961), 59-127.

[5] H.-J. Petzsche, On E. Borel's theorem, Math. Ann. 282 (1988), 299-313.

[6] T. J. Rivlin, Chebyshev Polynomials, Wiley, 1990.

[7] S. Roman, The formula of Faà di Bruno, Amer. Math. Monthly 87 (1980), 805-809.

[8] J. Schmets and M. Valdivia, Extension properties in intersections of non quasianalytic classes, Note Mat., to appear.

Institut de Mathématique

Facultad de Matemáticas

Université de Liège

Universidad de Valencia

Sart Tilman Bât. B 37

Dr. Moliner 50

B-4000 Liège 1, Belgium

E-46100 Burjasot (Valencia), Spain

E-mail: j.schmets@ulg.ac.be

Reçu par la Rédaction le 25.5.2005

Révisé le 24.10.2005 